FIMAT-8/95

\title{
Three-dimensional effects on the electronic structure of quasiperiodic systems
}

\author{
Enrique Maciá* and Francisco Domínguez-Adame \\ Departamento de Física de Materiales, Facultad de Físicas, Universidad Complutense, \\ E-28040 Madrid, Spain
}

\begin{abstract}
We report on a theoreticl study of the electronic structure of quasiperiodic, quasi-one-dimensional systems where fully three dimensional interaction potentials are taken into account. In our approach, the actual physical potential acting upon the electrons is replaced by a set of nonlocal separable potentials, leading to an exactly solvable Schrödinger equation. By choosing an appropriate trial potential, we obtain a discrete set of algebraic equations that can be mapped onto a general tight-binding-like equation. We introduce a Fibonacci sequence either in the strength of the on-site potentials or in the nearest-neighbor distances, and we find numerically that these systems present a highly fragmented, self-similar electronic spectrum, which becomes singular continuous in the thermodynamical limit. In this way we extend the results obtained so far in one-dimensional models to the three-dimensional case. As an example of the application of the model we consider the chain
\end{abstract}

*Also at the Instituto de Estudios Interdisciplinares, El Guijo, Z4 Galapagar, E-28260 Madrid, Spain. 
polymer case.

Typeset using REVTEX 


\section{INTRODUCTION}

The study of transport properties of self-similar aperiodic structures has been a continuously growing research field during the last decade. On the one side, the remarkable discovery of the quasicrystalline phase [1]:2] gave rise to a considerably amount of theoretical work on transport properties of Fibonacci lattices, regarded as archetypical models of quasiperiodic systems in one dimension (1D) [3 9. On the other side, since the first growth of semiconducting quasiperiodic superlattices [10,11], it has been progressively realized that electronic systems arranged according to a substitution sequence should offer interesting possibilities for technological applications. In addition, recent progresses in diffusion-controlled aggregation on surfaces, allowing to growth 1D chains of atoms on a substrate [12], along with proposals of nanoscale devices based on atomic switching in atomic wires [13], span considerably the interest of the quasiperiodic order notion [14 to properly describe transport properties of quasiperiodic systems (QS) at the nanostructure level.

It is well known that, for 1D systems, quasiperiodicity leads to highly fragmented energy spectra that are Cantor sets with zero Lebesgue measure in the thermodynamical limit [15]. This exotic spectrum determines the existence of electron wave functions that are neither extended in the Bloch sense nor localized, but display dramatic fluctuations over the whole system. As a consequence, transport properties exhibit a peculiar behavior even when finite temperature effects are taken into account [16]. To the date, however, most of the theoretical work on the transport properties of QS breaks into two main kinds of 1D models, namely, discrete tight-binding models and continuous Kronig-Penney models. Albeit purely 1D models frequently render as adequate approximations to more complex three-dimensional (3D) systems, it is also true that low dimensional treatments could give rise to spurious phenomena that are not met in going to higher dimensions. Then the question as to whether results obtained for purely $1 \mathrm{D}$ systems can be extended to more realistic, fully $3 \mathrm{D}$ models becomes very appealing from both theoretical and technological points of view. In fact, if the purported fragmentation of the electronic spectrum of QS is destroyed by 3D effects, then 
theoretical results obtained so far about its related characteristic transport properties are of no practical relevance and possible technological application of these interesting systems fade away.

In this work we address the task of elucidating whether the remarkable properties of 1D QS are inherent to the low dimensionality of the models or, on the contrary, they should be expected in 3D systems as well. To this end, we introduce a completely general model to study electronic properties in 3D QS. Our approach is based on the so-called nonlocal (separable) potential (NLP) method, in which the actual potential at each site of an arbitrary system is replaced by a projective operator 17 19. As an interesting working example, we shall consider in detail the case of trans-polyacetylene. The different nonlinear excitations present in polymers in general, like solitons, polarons, and bipolarons are not included in the present study. If one is interested in the effect of these types of excitations, it would be necessary to resort to many-body Hamiltonians like the Su-Schrieffer-Heeger or the PeierlsHubbard ones (see Ref. [20] and references therein). On the other hand, what we are interested in is on 3D electron dynamics in quasiperiodic systems, and in showing them as much clear as possible. Thus, it is reasonable to focus only on linear excitations. The fact that we use parameters for trans-polyacetylene later is because we have obtained them in the framework of our nonlocal potential model with great accuracy [19], and therefore they are already available to present an example of the orders of magnitude to be expected in polymer applications. In this way we show that characteristic features of 1D QS are still present when 3D interaction potentials are considered. The rest of the paper is organized as follows. In Sec. II we present our model, summarize previous work of us [19] that is necessary for a better understanding of the present paper, and obtain exact expressions for the physical magnitudes of interest in the study of general QS. Afterwards, in Sec. III we undertake a detailed study of the electronic properties of 3D QS. We obtain numerical results demonstrating the existence of a highly-fragmented, self-similar electronic spectrum, being singular continuous in the thermodynamical limit. Section IV concludes the paper with a brief discussion on the physical relevance of the obtained results. 


\section{THE NLP APPROACH TO THE STUDY OF QUASIPERIODIC SYSTEMS}

The solution of the Schrödinger equation for multicentre interactions is of interest in condensed matter physics as well as in atomic and molecular physics. As it is well known, such solution is expected to involve enormous difficulties since, in most cases, prohibitively cumbersome calculations are required. During last years several methods have been proposed to solve this problem. Among them, the NLP approach is the natural generalization of the Kronig-Penney model to the 3D case. This method yields an exactly solvable Schrödinger equation from which the electron energy can be readily obtained without tedious and elaborated calculations. What is more important, it is always possible to find a NLP (or a sum of them) able to reproduce any set of given electronic states [21] and, consequently, there is no theoretical limitation to the numerical accuracy with which physical results can be obtained. We shall open this section with a brief account of the general aspects of the NLP approach and, subsequently, we will apply it to the description of QS.

\section{A. Schrödinger equation for NLP}

The Schrödinger equation for NLP reads as follows [19 (we take $\hbar=m=1$ hereafter)

$$
\left(\mathbf{p}^{2}-2 E\right) \psi(\mathbf{r})=\sum_{k} \lambda_{k} V\left(\left|\mathbf{r}-\mathbf{R}_{k}\right|\right) \int d^{3} r^{\prime} V\left(\left|\mathbf{r}^{\prime}-\mathbf{R}_{k}\right|\right) \psi\left(\mathbf{r}^{\prime}\right)
$$

where $\mathbf{R}_{k}$ denotes the position of each atomic site and $\lambda_{k}$ is the corresponding coupling constant. It is usual to assume, as a first approximation, that the potential function $V$ is spherically symmetric, although other symmetries can be also considered within the NLP approach. By Fourier transforming we have

$$
\psi(\mathbf{p})=\left(\frac{1}{p^{2}-2 E}\right) \sum_{k} \lambda_{k} V(p) \exp \left(-i \mathbf{p} \cdot \mathbf{R}_{k}\right) \chi_{k}
$$

where

$$
\chi_{k}=\int d^{3} p V^{*}(p) \exp \left(i \mathbf{p} \cdot \mathbf{R}_{k}\right) \psi(\mathbf{p}) .
$$


Here $\psi(\mathbf{p})$ and $V(p)$ denote the Fourier transforms of $\psi$ and $V$, respectively. The coefficients $\chi_{k}$ are related to the wave function in real space, and we will discuss their meaning once we have specified the potential $V(p)$. Inserting (2) in (3) and performing the angular integration we obtain the following set of algebraic equations

$$
\chi_{k}=4 \pi \sum_{j} \lambda_{j} \int_{0}^{\infty} d p \frac{p^{2}|V(p)|^{2}}{p^{2}-2 E} \frac{\sin p R_{k j}}{p R_{k j}} \chi_{j},
$$

where $R_{k j} \equiv\left|\mathbf{R}_{k}-\mathbf{R}_{j}\right|$ and it is understood that the factor $\left(\sin p R_{k j}\right) / p R_{k j}$ is replaced by 1 when $k=j$.

\section{B. Application to quasi-one-dimensional lattices}

The set of algebraic equations given in (4) provide a completely general solution of the Schrödinger equation for multicentre interactions in three dimensions, as soon as the shape of the potential $V(p)$ is specified. Hence, the crucial point within the NLP approach is to choose an appropriate potential that reproduces the observed energy values for the physical system being considered. In this work we will be concerned with quasi-one-dimensional systems where an array of 3D potentials are arranged along a straight line. We have shown that the electronic band structure of quasi-one-dimensional polymers, such as trans-polyacetylene, can be quite accurately obtained making use of this approach and taking surface $\delta$-function interactions of the form 19

$$
V(r)=\frac{1}{r^{2}} \delta(r-R), \quad V(p)=\sqrt{\frac{2}{\pi}} \frac{\sin p R}{p R} .
$$

Physically this potential describes a highly localized force field which vanishes everywhere except on a spherical shell of radius $R$ around the lattice site. In this particular case Eq. (四) takes the form

$$
\chi_{k}=\frac{\lambda_{k}}{R^{2}} A(E) \chi_{k}+\sum_{j \neq k} \frac{\lambda_{j}}{R^{2}} B_{k j}(E) \chi_{j},
$$

where for brevity we have defined 


$$
\begin{aligned}
A(E) & =\frac{2 \pi}{\kappa}[1-\exp (-2 \kappa R)] \\
B_{k j}(E) & =\frac{2 \pi \exp \left(-\kappa R_{k j}\right)}{\kappa^{2} R_{k j}}(\cosh 2 \kappa R-1) .
\end{aligned}
$$

The corresponding integrals have been performed for the case of interest, namely $E<0$, and then $\kappa \equiv \sqrt{-2 E}$ is a real parameter. Notice that the larger the distance between site $k$ and $j$, the smaller the corresponding coefficient $B_{k j}(E)$. In other words, such coefficients are rapidly decreasing functions of $R_{k j}$ provided that $\kappa$ is not very small (deep potentials). This is usually a good approximation in most cases of practical interest and, for these systems, it is reasonable to assume that only nearest-neighbor interactions are significant. Thus

$$
\chi_{k}=\frac{\lambda_{k}}{R^{2}} A(E) \chi_{k}+\frac{\lambda_{k+1}}{R^{2}} B_{k k+1}(E) \chi_{k+1}+\frac{\lambda_{k-1}}{R^{2}} B_{k k-1}(E) \chi_{k-1} .
$$

At this point it becomes convenient to provide some physical insight into the coupling constants $\lambda_{j}$ and, to this end, we must consider the single site case for a moment. When only one atom at $\mathbf{R}_{k}=0$ is present in the system, the NLP Schrödinger equation reduces to

$$
\left(\mathbf{p}^{2}-2 E\right) \psi(\mathbf{r})=\lambda_{k} V(r) \int d^{3} r^{\prime} V\left(r^{\prime}\right) \psi\left(\mathbf{r}^{\prime}\right)
$$

from which we get

$$
\frac{1}{4 \pi \lambda_{k}}=\int_{0}^{\infty} d p \frac{p^{2}|V(p)|^{2}}{p^{2}-2 E_{k}} .
$$

This relation enables us to link the coupling constant of any arbitrary site of the system with its single bound state energy level $E_{k}=-\kappa_{k}^{2} / 2$, an experimentally measurable quantity. Making use of expressions (5) and (10) we get $\lambda_{k} A\left(E_{k}\right) / R^{2}=1$. For short-ranged potentials we can expand $A\left(E_{k}\right)$ in powers of $R$ obtaining

$$
\lambda_{k} \sim \frac{R}{4 \pi}\left(1+\kappa_{k} R\right)
$$

In this way we can estimate the most appropriate values of the coupling constants from experimental data. Now we go back to our general treatment. Inserting (11) in (8) and taking the limit $R \rightarrow 0$ in such a way that $E_{k}$ remains constant, we obtain the following tight-binding equation for the coefficients $\chi_{k}$ 


$$
\left(\kappa-\kappa_{k}\right) \chi_{k}=\frac{\exp \left(-\kappa R_{k k+1}\right)}{R_{k k+1}} \chi_{k+1}+\frac{\exp \left(-\kappa R_{k-1}\right)}{R_{k k-1}} \chi_{k-1} .
$$

Note that the corresponding transfer integrals decrease exponentially on the distance between nearest-neighbors, as it should be expected, and their functional form is typical of s-orbitals. In addition they depend explicitly on the electron energy. Therefore, at this stage of our analysis, the mathematical treatment of the system remains fully $3 \mathrm{D}$ although the system is quasi-one-dimensional. Before proceeding, we wish to clarify the physical meaning of $\chi_{k}$. From their definition in Eq. (3) and the Parseval identity we have

$$
\chi_{k}=\int d^{3} r V(r) \psi\left(\mathbf{r}+\mathbf{R}_{k}\right)
$$

with $V(r)=r^{-2} \delta(r-R)$. In the limit $R \rightarrow 0$ one gets $V(r) \rightarrow r^{-2} \delta(r)=\delta(\mathbf{r})$. Therefore, in this limiting case

$$
\chi_{k}=\psi\left(\mathbf{R}_{k}\right) .
$$

Thus we see that $\chi_{k}$ is nothing but the value of the electron wave function at site $\mathbf{R}_{k}$, which is of course the quantity of interest.

\section{The NLP approach for quasiperiodic quasi-one-dimensional systems}

We now proceed introducing quasiperiodicity in our lattice model. This can be accomplished in two different ways. On the one hand, we can assume the nearest-neighbor distance to be constant and consider two kind of different basic units, say A and B, arranged quasiperiodically along the chain. Examples of these systems may be provided by binary Fibonacci quasicrystals or multilayered heterostructures. On the other hand, we can assume all the sites to be the same and vary the distance between them in a quasiperiodic fashion. In this case, the sites can be occupied by either single atoms (self-assembled aggregates), molecules (homopolymers) or an assembly of crystalline monolayers (superlattices). When applied to the tight-binding Eq. (12), the first option corresponds to on-site models, whereas the second one describe transfer models. 
During the last years several quasiperiodic arrangements, based on the application of substitution rules, have been considered in the literature. In this work we will focus on the Fibonacci arrangement as a canonical example of QS which has been experimentally probed in a variety of situations [22 25] in the knowledge that our treatment can be similarly applied to other self-similar aperiodic realizations in a straightforward manner. In general, a Fibonacci chain of order $l$ is generated from two basic units $A$ and $B$ by successive applications of the substitution $A \rightarrow A B$ and $B \rightarrow A$ yielding a sequence of the form $A B A A B A B A \ldots$ This sequence comprises $F_{l-1}$ elements $A$ and $F_{l-2}$ elements $B$, where $F_{l}$ is the $l$ th Fibonacci number given by the recurrent law $F_{l}=F_{l-1}+F_{l-2}$ with $F_{0}=F_{1}=1$. As $l$ increases the ratio $F_{l-1} / F_{l}$ converges toward $\tau=(\sqrt{5}-1) / 2$, which is known as the inverse golden mean.

The electron dynamics of this system can be conveniently studied by means of the wellknown transfer-matrix techniques. To this end, we cast Eq. (12) into the matrix form

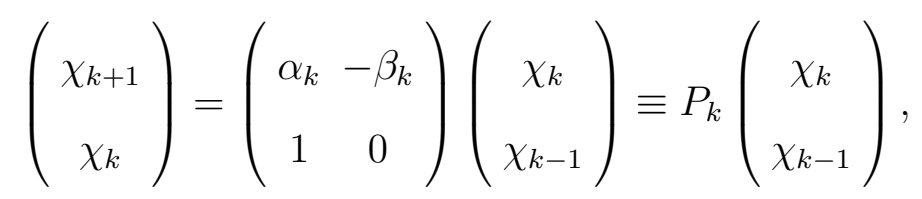

where $\alpha_{k}=\left(\kappa-\kappa_{k}\right) R_{k k+1} \exp \left(\kappa R_{k k+1}\right)$ and $\beta_{k}=\left(R_{k k+1} / R_{k k-1}\right) \exp \left(\kappa R_{k k+1}-\kappa R_{k k-1}\right)$. The transfer matrix of the whole system is then found as

$$
T(N)=\prod_{k=N}^{1} P_{k},
$$

which relates the wave function at both edges of the system. Taking into account the fact that $T(N)=P_{N} T(N-1)$ with $T(0)$ the $2 \times 2$ unity matrix, we find the following recurrence relations to compute the transfer-matrix elements

$$
\begin{aligned}
& T_{11}(n)=\alpha_{n} T_{11}(n-1)-\beta_{n} T_{11}(n-2), \\
& T_{12}(n)=\alpha_{n} T_{12}(n-1)-\beta_{n} T_{12}(n-2), \\
& T_{21}(n)=T_{11}(n-1), \\
& T_{22}(n)=T_{12}(n-1), \quad n=2,3 \cdots N .
\end{aligned}
$$


with the initial conditions $T_{i j}(0)=\delta_{i j}, T_{11}(1)=\alpha_{1}, T_{12}(1)=-\beta_{1}, T_{21}(1)=1$ and $T_{22}(1)=0$. Here $N$ is a Fibonacci number indicating the number of basic units of the system. Some physically relevant magnitudes can be readily obtained from the transfer-matrix $T(N)$. Although the information that anyone of these magnitudes can provide isolately is not conclusive, when grouped together they produce a clear picture on the nature of the electronic states [26]. The Lyapunov coefficient, representing the growth rate of the wave function, is nothing but the inverse of the localization length. As a consequence, delocalization of

electronic wave function is seen through the decrease of this parameter. It can be computed as [27]

$$
\Gamma(E)=\left(\frac{1}{N}\right)\left(T_{11}^{2}(N)+T_{12}^{2}(N)+T_{21}^{2}(N)+T_{22}^{2}(N)\right)
$$

The fragmentation scheme and the number of states in each subband is characterized by means of the integrated density of states (IDOS) per unit length. Finally, considering periodic boundary conditions at both edges of the system, the following condition for an energy to be in an allowed band is obtained

$$
\left(\frac{1}{2}\right)|\operatorname{Tr}[T(N)]| \leq 1
$$

Making use of this condition the equivalent bandwidth (defined below) of the spectrum can be readily determined and, from its scaling properties with the system size, we can infer the spectral signature of the electronic spectrum. All these expressions are very simple and suitable for an efficient numerical treatment. We will now evaluate them for several interesting cases.

\section{RESULTS AND DISCUSSIONS}

For convenience, we shall describe the results obtained for transfer and on-site models separately. 


\section{A. Quasiperiodic transfer systems}

As an illustrative example we shall consider a quasi-one-dimensional array of $\mathrm{CH}$ units roughly representing the trans-polyacetylene chain. There are two parameters that can be varied in a transfer model, namely, the values of the short, $R_{S}$, and long, $R_{L}$, bond lengths between neighboring units. Two different sets of short and long alternating bonds have been considered describing the extreme cases of weak $\left(R_{L}=1.43 \AA, R_{S}=1.36 \AA\right)$ and strong $\left(R_{L}=1.54 \AA, R_{S}=1.34 \AA\right)$ polymer stretching [28]. Making use of previous results 19] we can estimate the range of on-site energies able to reproduce the experimentally observed band edges in trans-polyacetylene depending on its stretching state. In this way we obtain $E_{0} \sim-6.0 \mathrm{eV}$ to be an appropriate on-site energy on the average. Chains containing up to $\mathrm{N}=F_{15}=987 \mathrm{CH}$ units have been studied numerically making use of the expressions derived in the previous section. In Fig. 1 we show the fragmentation of the $\pi$-band as a function of the Fibonacci number obtained from condition (19) in the case of strong stretching. Only short approximants of the Fibonacci sequence are displayed since, on increasing $\mathrm{N}$, the spectrum becomes so fragmented that it becomes very difficult to observe minor features in the plot. We have carefully analysed, however, spectra up to $N=987$ in order to determine the spectrum splitting pattern. In this way we have confirmed that the single band corresponding to the periodic case splits into four main subbands which further split into smaller subsubbands. As soon as a quasiperiodic arrangement of the conjugated bonds is introduced, the position and widths of the main subbands of the spectrum converge rapidly to stable values on increasing the polymer length. This behavior implies that the global structure of the electronic spectrum can be obtained, in practice, by considering very short approximants to the infinite, strictly quasiperiodic realization. In fact, this is the case for system size as short as $N=F_{8}=34$ in the considered model. This characteristic has been referred to as the asymptotic stability of the spectrum [16].

From a statistical point of view, valuable information can be extracted from the way in which the electronic spectrum splits on increasing the number of units in the chain. Our 
previous experience in the study of 1D systems indicates that the IDOS per unit length is a powerful tool to study the occupation of the different subbands of the fragmented spectrum. In Fig. 2 we present a typical IDOS evaluated by means of node counting. Its stair-case shape provides additional support on the self-similar splitting scheme just reported on. In fact, the overall tetrafurcation pattern of the spectrum is characterized by the presence of four main subbands, labeled $a, b, c$, and $d$ in Fig. 2, separated by well-defined plateaus. Inside each main subband the fragmentation proceeds obeying a trifurcation scheme which can be quantitatively described as follows. The number of subsubbands in each main subband is given by $N_{a}=F_{l-2}, N_{b}=F_{l-3}, N_{c}=F_{l-4}$, and $N_{d}=F_{l-3}$ in agreement with distribution rules reported for 1D tight-binding models [16,29. We define the occupation of a main subband as the ratio between the number of states in that subband and the total number of electronic states, this is to say, $q_{i}=N_{i} / N(i=a, b, c, d)$. Since each site in the carbon backbone contributes with one electronic state to the energy spectrum, this occupation can be directly measured from the heights of the characteristic steps appearing in the IDOS. In this way we have determined that $q_{a}=\tau^{2}, q_{b}=\tau^{3}, q_{c}=\tau^{4}$, and $q_{d}=\tau^{3}$, within an error less than $0.1 \%$, for a wide collection of system realizations. it is interesting to note that these occupations correspond to a nonequilibrium level distribution for the system at zero temperature, in agreement with previous results for 1D QS [14].

\section{B. Quasiperiodic on-site systems}

There are two parameters that can be varied in an on-site model, namely, the bound state levels of the composing units $E_{A}$ and $E_{B}$. For the sake of illustration we shall consider a quasi-one-dimensional array of $\mathrm{CH}$ units describing chemically doped trans-polyacetylene. By this we mean that the native polymer has been subjected to a chemical process consisting of either a charge transfer reaction by oxidizing or reducing agents or an acidic treatment in which protons attach to certain sites of the chain. In our model unchanged polymer units will be located in sites $A$ whereas units doped in this way are placed quasiperiodically at 
sites $B$. Assuming an uniform carbon-carbon bond length of $L=1.39 \AA$ [28]. we obtain that $E_{A} \sim-6.0 \mathrm{eV}$ reproduces quite well the observed band edges in trans-polyacetylene [19]. On the other side, the value of $E_{B}$, which describes the effect of doping on the different units, will be varied in the range from $-6.0 \mathrm{eV}$ up to $-12.0 \mathrm{eV}$ in order to ascertain its influence on the electronic structure of the system.

Making use of the value $R_{k \pm 1}=L$ and defining $\rho \equiv \kappa L$ and $\rho_{k} \equiv \kappa_{k} L$ for the sake of brevity, we can express the tight-binding equation (12) in the form

$$
\left(\rho-\rho_{k}\right) e^{\rho} \chi_{k}=\chi_{k k+1}+\chi_{k k-1}
$$

We have checked that only one band is placed in the negative-energy interval in the case of native trans-polyacetylene with an uniform carbon-carbon bond length and that, when doping is uniformly introduced, this band becomes deeper and narrower on decreasing the value of the on-site energy associated to the doped sites. The general behavior of the $\pi$-band as a function of this on-site energy is shown in Fig. 3. Deviation from native polymer state can be quantitatively measured by means of the ratio $\alpha=E_{B} / E_{A}$, hereafter called doping parameter. The more distant is $\alpha$ from unity, the more important is the effect of doping.

Using of condition (19), we have found that, as soon as the native polymer is chemically modified at sites arranged quasiperiodically, the $\pi$-band splits into four main subbands which further split into smaller subsubbands. The observed splitting hierarchy is completely analogous to that shown in Fig. 1. A characteristic feature of Fibonacci systems, which has been thoroughly purported in the study of $1 \mathrm{D}$ systems, concerns the self-similar pattern exhibited by their energy spectra. In Fig. 4 we show a typical plot of the Lyapunov coefficient as a function of the electron energy. In this plot the maxima indicate the position of the minigaps determining the spectrum fragmentation discussed before. By inspecting this plot, it is clear that the whole spectrum corresponding to a short Fibonacci approximant is mapped onto a small portion of the spectrum.

It has been rigorously shown that, in the thermodynamical limit, 1D Fibonacci systems present a singular continuous electronic spectrum [30]. In order to estimate the spectral 
type associated to our system, we have computed the so-called equivalent bandwidth $S$, defined as the sum of all allowed subbands. As can be expected from the Cantor-like nature of Fibonaccian spectra, $S$ vanishes as the polymer size increases according to a power law of the form $S=F_{l}^{-\beta(\alpha)}$. In Fig. 5 we show the dependence of the equivalent bandwidth on the polymer size for different values of the doping parameter $\alpha$. We have confirmed that $S$ obeys a power law behavior even when high doping parameters are considered (up to $\alpha=2$ ). According to earlier results [31], such a power-law scaling is characteristic of a singular continuous spectrum for which all the wave functions are critical, i. e., regarding localization properties the functions are neither exponentially localized nor extended in the Bloch sense. Hence we are led to the conclusion that 3D effects do not change the critical nature of the electronic states in QS. To get more information on how the electronic spectra behaves in approaching the thermodynamical limit we undertook the study of the dependence of the critical exponent $\beta$ on the ratio $\alpha=E_{B} / E_{A}$ with a fixed value $E_{A}=-6.0 \mathrm{eV}$. Results shown in Fig. 6 evidence that $\beta$ raises linearly with $\alpha$, hence suggesting that the electronic spectrum is fragmented more rapidly on increasing the chemical diversity of the chain. This is to be expected since the fragmentation of the $\pi$-band arises as a consequence of loss of quantum coherence, and this effect is enhanced as the on-site energies $\left(E_{A}\right.$ and $\left.E_{B}\right)$ become more and more different due to the doping process.

\section{CONCLUSIONS}

In this paper we have considered electron dynamics in quasi-one-dimensional Fibonacci systems. Our procedure, based on the NLP approach, allows us to carry out a fully three dimensional analysis of the considered system. It is important to realize that this technique can be applied to any desired level of precision. In addition, the exact solution can be found for an arbitrary NLP, as we actually demonstrated [see Eq. (四)] by means of the Fourier transform which, in turn, is completely equivalent to the use of the Green function formalism. We have considered surface $\delta$-function interactions with vanishing radius since this potential 
gives very accurate results in the study of trans-polyacetylene [19]. We have focused on the analysis of quasiperiodic arrangements where atoms are placed in a straight line. Nevertheless, the model can be easily extended to other possible geometric configurations of interest as, for example, zig-zag chains whose $s p^{2}$ hybridization geometry is explicitly considered, or branched atomic wires as those obtained for diffusion-controlled aggregates.

In this work we demonstrate that all those features reported as to being typical of quasiperiodic (Fibonacci) systems in one dimension can be extended to the three dimensional case as well. In this way we provide substantial support to the interest of these novel systems from an applied viewpoint. In particular we wish stress the fact that the most characteristic property of electronic energy spectrum of QS, namely, its highly fragmented self-similar structure, is expected to be observed also in more realistic (3D) realizations. This result strongly suggests that the peculiar nature of the spectra associated to quasiperiodic systems has nothing to do with the number of spatial dimensions considered in the interaction potential but it is directly related to the topological order implied by the way in which the basic units are arranged in the system. A further elaboration of this point, dealing with three dimensional quasiperiodic Schrödinger Hamiltonian operators from a mathematical point of view will be, therefore, very appealing.

Albeit we have focused on a particular realization, trans-polyacetylene, attending to the practical interest of this system, our main results are very general in character and, in fact, encompass a broad class of quasiperiodically ordered systems, as we have discussed previously. As a consequence, we can confidently drawn the following conclusions. In the first place, it is worth noticing that the self-similar fragmentation of the spectrum is a robust property of 3D QS. Indeed, we have confirmed it in two different scenarios, namely, extreme cases of stretched bonding (transfer models) and wide range of chemical doping (on-site models). In both cases the hierarchy of the splitting spectrum pattern remains unchanged and the gap labelling ordering preserved. In the second place, we observe that the asymptotic stability of the electronic spectrum, which was previously reported in the study of quasiperiodic superlattices [16]. can be extended to a wide class of QS. This result 
has important consequences from a practical viewpoint as it indicates that typically fractal features can be observed in the transport properties of relatively short QS, which are within the domain of actual technological capabilities. In the third place, we have shown that the equivalent bandwidth of the electronic spectrum can be modified in a precise way by properly choosing the sample size and the chemical diversity, hence allowing for a certain kind of engineering of the related transport properties. Finally, we confirm that the systems which we are dealing with deserve a proper position in the orderings of matter by their own right. In fact, in a recent work [14] we have reported that 1D QS are able to encode more information, in the Shannon sense, than other usual orderings of matter are able to do. This feature is intimately related to the fact that QS describe far from thermodynamical equilibrium systems. In this work we provide substantial support to this view, since we are considering a polymer which is not in the equilibrium state described by an alternating pattern of the conjugated bonds. Instead, we are dealing with a polymer with either a quasiperiodic arrangement of short and long bonds or a quasiperiodic sequence of chemically doped sites. In both cases we are describing systems which are not in its state of minimum energy. Therefore, it is not surprising that the corresponding IDOS display reversed level distribution at zero temperature (see Fig. 2), a typical feature of nonequilibrium systems. To the best of our knowledge the experimental realization of such a kind of polymers has not been reported in the literature. In this sense, we expect the theoretical results we report in this work could receive further attention by experimental researchers.

\section{ACKNOWLEDGMENTS}

This work is supported by CICYT through project MAT95-0325. 


\section{REFERENCES}

[1] D. Shechtman, I. Blech, D. Gratias, J. W. Cahn, Phys. Rev. Lett. 56, 1951 (1984).

[2] A. I. Goldman and R. F. Kelton, Rev. Mod. Phys. 65, 213 (1993).

[3] B. Sutherland and M. Kohmoto, Phys. Rev. B 36, 5877 (1987).

[4] D. Würtz, M. P. Sorensen, and T. Schneider, Helv. Phys. Acta 61, 345 (1988).

[5] M. Goda and H. Kubo, J. Phys. Soc. Jpn. 58, 2109 (1989).

[6] M. Severin, M. Dulea, and R. Riklund, J. Phys. Condens. Matter 1, 8851 (1989).

[7] R. B. Capaz, B. Koiller, and S. L. A. de Queiroz, Phys. Rev. B 42, 6402 (1990).

[8] M. E. J. Newman and R. B. Stinchcombe, Phys. Rev. B 43, 1183 (1991).

[9] F. Domínguez-Adame and A. Sánchez, Phys. Lett. A 159, 153 (1991).

[10] R. Merlin, K. Bajema, R. Clarke, F. -Y. Juang, and P. K. Bhattacharya, Phys. Rev. Lett. 55, 1768 (1985).

[11] J. Todd, R. Merlin, R. Clarke, K. M. Mohanty, and J. D. Axe, Phys. Rev. Lett. 57, 1157 (1986).

[12] H. Röder, E. Hahn, H. Brune, J. -P. Bucher, and K. Kern, Nature 366, 141 (1993).

[13] Y. Wada, T. Uda, M. Lutwyche, S. Kondo, and S. Heike, J. Appl. Phys. 74, 7321 (1993).

[14] E. Maciá, F. Domínguez-Adame, and A. Sánchez, Phys. Rev. E 50, R679 (1994).

[15] J. M. Luck, Phys. Rev. B 39, 5834 (1989).

[16] E. Maciá, F. Domínguez-Adame, and A. Sánchez, Phys. Rev. B 49, 9503 (1994).

[17] B. W. Knight and G. A. Peterson, Phys. Rev. 132, 1085 (1963).

[18] P. R. Sievert and M. L. Glasser, Phys. Rev. B 7, 1265 (1973). 
[19] F. Domínguez-Adame, B. Méndez, E. Maciá, and M. A. González, Mol. Phys. 74, 1065 (1991).

[20] A. J. Heeger, S. Kivelson, J. R. Schrieffer, and W. P. Su, Rev. Mod. Phys. 60, 781 (1988).

[21] M. L. Glasser, Surf. Sci. 64, 141 (1977).

[22] F. Laruelle and B. Etienne, Phys. Rev. B 37, 4816 (1988).

[23] M. Nakayama, H. Kato, and S. Nakashima, Phys. Rv. B 36, 3472 (1987).

[24] K. Kono, S. Nakada, Y. Narahara, and Y. Ootuka, J. Phys. Soc. Jpn. 60, 368 (1991).

[25] D. Tuet, M. Potemski, Y. Y. Wang, J. C. Maan, L. Tapfer, and K. Ploog, Phys. Rev. Lett. 66, 2128 (1991).

[26] A. Sánchez, E. Maciá, and F. Domínguez-Adame, Phys. Rev. B 49, 147 (1994).

[27] R. E. Borland, Proy. R. Soc. London 274A, 529 (1963).

[28] P. M. Grant and I. P. Batra, Solid State Commun. 29, 225 (1979).

[29] Y. Liu nad R. Riklund, Phys. Rev. B 35, 6034 (1987).

[30] A. Bovier and J. -M. Ghez, Commun. Math. Phys. 158, 45 (1993).

[31] M. Kohmoto, Phys. Rev. Lett. 51, 1198 (1983). 


\section{FIGURES}

FIG. 1. Allowed subbands as a function of the Fibonacci order $l$ for a QS polymer with $R_{L}=1.54 \AA, R_{S}=1.34 \AA$ and $E=-6.0 \mathrm{eV}$

FIG. 2. IDOS versus energy for a QS polymer with $L_{A}=1.54 \AA, L_{B}=1.34 \AA, E=-6.0 \mathrm{eV}$ and $N=F_{15}=987$. The main subbands are labelled by $a, b, c$ and $d$ along with their respective occupations. See the text for further details.

FIG. 3. Allowed energies (shaded region) as a function of the on-site energy in the native trans-polyacetylene. The carbon-carbon bond length is $L=1.39 \AA$.

FIG. 4. (a) Lyapunov coefficient as a function of energy for a QS polymer with $L=1.39 \AA$, $E_{A}=-6.0 \mathrm{eV}$ and $E_{B}=-7.0 \mathrm{eV}$. (b) An enlarged view of one of the main subbands illustrating its self-similar nature.

FIG. 5. Equivalent bandwidth $S$ as a function of the system size $N$ for $E_{A}=-6.0 \mathrm{eV}$ and different values of $E_{B}$, indicated on each curve. The carbon-carbon bond length is $L=1.39 \AA$.

FIG. 6. Critical exponent $\beta$ as a function of the doping ratio $\alpha=E_{B} / E_{A}$ with $E_{A}=-6.0 \mathrm{eV}$. The carbon-carbon bond length is $L=1.39 \AA$. 